\title{
Early prediction of poor outcome in patients with acute asthma in the emergency room
}

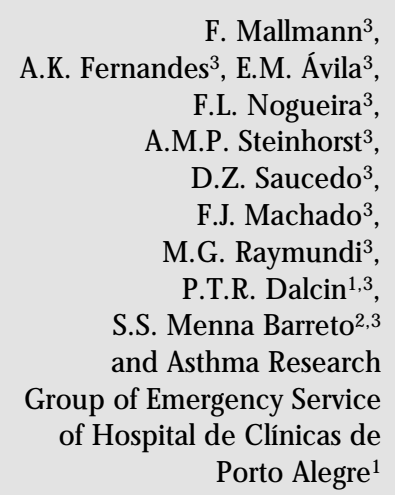

F. Mallmann ${ }^{3}$,

A.K. Fernandes'3, E.M. Ávila,

F.L. Nogueira ${ }^{3}$,

A.M.P. Steinhorst ${ }^{3}$,

D.Z. Saucedo ${ }^{3}$,

F.J. Machado ${ }^{3}$,

M.G. Raymundi ${ }^{3}$,

P.T.R. Dalcin ${ }^{1,3}$,

S.S. Menna Barreto 2,3

and Asthma Research

Group of Emergency Service

of Hospital de Clínicas de

Porto Alegre ${ }^{1}$

\author{
Services of ${ }^{1}$ Emergency and 2Pneumology, Hospital de Clínicas de Porto Alegre, \\ ${ }^{3}$ Faculdade de Medicina, Universidade Federal do Rio Grande do Sul, \\ Porto Alegre, RS, Brasil
}

\section{Correspondence}

P.T.R. Dalcin

Av. Cristóvão Colombo, 462/306

Bloco B

90560-000 Porto Alegre, RS

Brasil

Fax: + 55-51-330-0521

E-mail: dalcin@ conex.com.br

Research supported by FAPERGS, Programa de Iniciação Científica da UFRGS, and FIPE-HCPA.

Received May 22, 2001 Accepted October 9, 2001

\section{Abstract}

Early identification of patients who need hospitalization or patients who should be discharged would be helpful for the management of acute asthma in the emergency room. The objective of the present study was to examine the clinical and pulmonary functional measures used during the first hour of assessment of acute asthma in the emergency room in order to predict the outcome. We evaluated 88 patients. The inclusion criteria were age between 12 and 55 years, forced expiratory volume in the first second below $50 \%$ of predicted value, and no history of chronic disease or pregnancy. After baseline evaluation, all patients were treated with $2.5 \mathrm{mg}$ albuterol delivered by nebulization every $20 \mathrm{~min}$ in the first hour and $60 \mathrm{mg}$ of intravenous methylprednisolone. Patients were reevaluated after $60 \mathrm{~min}$ of treatment. Sixty-five patients (73.9\%) were successfully treated and discharged from the emergency room (good responders), and 23 (26.1\%) were hospitalized or were treated and discharged with relapse within 10 days (poor responders). A predictive index was developed: peak expiratory flow rates after $1 \mathrm{~h} \leq 0 \%$ of predicted values and accessory muscle use after $1 \mathrm{~h}$. The index ranged from 0 to 2 . An index of 1 or higher presented a sensitivity of 74.0, a specificity of 69.0 , a positive predictive value of 46.0 , and a negative predictive value of 88.0 . It was possible to predict outcome in the first hour of management of acute asthma in the emergency room when the index score was 0 or 2 .

\section{Introduction}

Acute asthma is a very common medical emergency and is responsible for increasing hospitalization and death rate (1). Patients with severe acute asthma are usually hospi-
Key words

- Asthma

- Outcome

- Index

- Prognosis

- Emergency medicine talized only after one attempt at therapy in the emergency room (ER). Consequently, many patients spend long hours in a busy ER before a decision to hospitalize or discharge them is made (2). In spite of this, relapse rates in patients treated and discharged from 
the ER remain as high as 26 to $30 \%$, requiring further ER care $(2,3)$. The early identification of patients who need hospitalization or patients who should be discharged would be helpful for the management of acute asthma in the ER (4).

Clinical evidence demonstrates that the first hour of management of acute asthma in the ER entails crucial decisions that could determine the clinical outcome (5). Current guidelines recommend an initial treatment with $2.5 \mathrm{mg}$ albuterol by nebulization every $20 \mathrm{~min}$ for $60 \mathrm{~min}$ (three doses) (6). Patients with less airway inflammation usually demonstrate a good response to therapy and can be discharged with close follow-up. Conversely, the failure of initial therapy to improve expiratory flow predicts a more severe course and the need for hospitalization (4). The response to initial treatment in the ER is a better predictor of the need for hospitalization than is the severity of an exacerbation at presentation (7).

In spite of a variety of clinical and laboratory measures currently used to assess acute asthma severity, no single criterion has been found to reliably predict outcome (8). Rodrigo and Rodrigo (8) used multivariate factor analysis to determine that asthma appears to be multidimensional and that most asthma measures reflect a much smaller number of underlying dimensions. This justifies the use of a relatively small number of predictors in the assessment of outcome after episodes of acute asthma.

The objective of the present study was to examine the clinical and pulmonary function measures used in the first hour of assessment of acute asthma in the ER to predict the outcome of acute episodes.

\section{Material and Methods}

\section{Design}

We studied a cohort of patients presenting exacerbated asthma in the adult ER of a large-tertiary care hospital, from May to November 2000 , to examine the clinical and functional measures used in the first hour of assessment in the ER to predict the outcome of acute asthma. We prospectively followed the outcome of these patients within the next 10 days. The protocol was reviewed and approved by the Ethics and Research Committee of Hospital de Clínicas de Porto Alegre, and informed consent for participation in the study was obtained from patients or parents.

\section{Subjects}

We studied 88 consecutive patients (25 men and 63 women) who appeared for treatment of acute asthma to the adult ER of Hospital de Clínicas, Porto Alegre, RS, Brazil. Patients were eligible to participate if they had a clinical diagnosis of asthma confirmed by an emergency physician in accordance with the criteria of the American Thoracic Society (9). The inclusion criteria were: 1) age between 12 and 55 years, 2) forced expiratory volume in the first second $\left(\mathrm{FEV}_{1}\right)$ below $50 \%$ of predicted value, and 3 ) no history of chronic cough, emphysema, cardiac, hepatic, renal or other lung disease, or pregnancy. Patients were excluded from the study if they were unable to perform the diagnostic tests proposed by the study, or if they had withdrawn consent during the study.

\section{Measures and procedures}

On admission, all patients were assessed by an emergency physician and by the project member. A short history was recorded on standardized forms, including age, sex, the presence of symptoms of dyspnea, cough, the feeling of thoracic constriction, the duration of the present attack, smoking habits, passive smoking, and the present and past asthma medications. Height and weight were recorded. The physical examination in a sit- 
ting position included determination of pulse rate, respiratory rate, pulsus paradoxus, central cyanosis, accessory muscle use, supraclavicular or intercostal retractions, inability to speak and wheezing. Pulsus paradoxus was judged to be present when a value of 15 $\mathrm{mmHg}$ or more was measured. Accessory muscle use was defined as visible and palpable contraction of the sternocleidomastoid muscles during the inspiratory phase of tidal breathing.

Spirometry was performed with the subjects in the sitting position using computerized equipment (Collins Survey II Spirometer interfaced to a Collins Eagle II Microprocessor, Warren E. Collins, Inc., Boston, MA, USA) to measure the $\mathrm{FEV}_{1}$, the forced vital capacity and the peak expiratory flow rates (PEFR). Three successive maximal expiratory curves were recorded at each assessment and the highest value was selected. The results were expressed in relation to predicted values (10). The arterial oxygen saturation was measured using a pulse oximeter and finger probe (Dx 2405/Oxímetro de pulso/Oxipleth, Dixtal, Manaus, AM, Brazil) with the patient breathing air and the value was noted when stable.

Patients were then started on treatment according to a standardized treatment protocol.

Physical examination (as already described), spirometry and pulse oximetry were repeated after $1 \mathrm{~h}$ of treatment supervised by the project member.

Patients were assessed for admission or discharge by an independent physician who acted on clinical judgment alone without knowledge of the data on lung function. Patient discharge from the ER was based on the following situations: when they were no longer dyspneic, when accessory muscle use abated, and when wheezing was judged minimal or resolved. The maximal duration of ER care was $24 \mathrm{~h}$. Decisions about hospitalization could be made before the time limit.

\section{Treatment protocol}

After initial assessment, all patients were treated with $2.5 \mathrm{mg}$ albuterol $(0.5 \mathrm{ml}$ of a $0.5 \%$ solution in $2.0 \mathrm{ml}$ normal saline), by continuous flow nebulization every $20 \mathrm{~min}$ for $60 \mathrm{~min}$ (three doses) followed by hourly treatments during the period of ER therapy.

A flow of $91 / \mathrm{min}$ of air compressed into the nebulizer generated the aerosol. This system has been characterized as producing a particle with a mass median aerodynamic diameter of $0.86 \mu \mathrm{m}$ and a geometric standard deviation of 0.89 (11). The subjects inhaled the aerosol for $5 \mathrm{~min}$ at their normal tidal volume while seated.

All patients also initially received $60 \mathrm{mg}$ methylprednisolone intravenously followed by $60 \mathrm{mg}$ every $6 \mathrm{~h}$, if needed. When the arterial oxygen saturation assessed by pulse oximetry was less than $92 \%, 1-21 / \mathrm{min}$ of supplemental oxygen was administered through a nasal cannula. Oral steroids were prescribed (a 7-day course of $40 \mathrm{mg}$ prednisone daily) for all patients who were discharged from the ER. Hospitalized patients received treatment according to the discretion of individual physicians.

\section{Follow-up}

The outcome was evaluated prospectively. The length of hospital stay and the need for medical intensive care, if any, were recorded for all hospitalized patients. All patients discharged from the ER were contacted 10 days later by telephone or by mail, or by a scheduled ambulatory visit with the project member, to determine the occurrence of relapse, if any. Relapse was defined as the need for further emergency care for asthma within the next 10 days.

Patients designated as good responders were those successfully discharged from the ER who did not require a visit to a hospital's ER or hospital admission for acute asthma treatment within the next 10 days. Patients 
designated as poor responders were those admitted to the hospital at the initial ER visit or those discharged from the ER who required a visit to a hospital's ER or hospital admission for acute asthma treatment within the next 10 days.

\section{Statistical analysis}

Data from good responders and poor responders were compared. Mean values $( \pm$ SD) were calculated for all variables in each group and examined for statistical significance by the Student $t$-test for normally distributed continuous variables and by the Mann-Whitney U-test for non-normally distributed continuous variables, or by the chisquare test with Yates correction for categorical variables. All probabilities reported were two-tailed. A P value of less than 0.05 was considered statistically significant.

Variables which were significant at the $\mathrm{P}<0.10$ level were entered into a multivariate logistic regression analysis. For this analysis we selected PEFR (baseline and after 1 $\mathrm{h}$ of treatment) as a pulmonary function test (variables with colinearity). Predictor variables from multivariate analysis were then entered into a forward stepwise program.

The variables that were statistically significant by multivariate analyses were selected. Each selected variable was then correlated with outcome to determine a single cutoff point for that variable that maximized predictive significance, using a receiver operating characteristic (ROC) curve. A predictor index for poor responders was then developed from these factors. In order to simplify the calculation of this index, a value of either 0 or 1 was assigned to each of the selected variables. These numerical values were summed to give a total index score of 0 to 2 according to the severity of the measures. As done for the analysis of individual variables, a ROC curve was constructed by varying the operating cutoff point of the index. Index sensitivity, specificity, and positive and negative predictive values were then calculated.

\section{Results}

Of the 344 patients who appeared at the adult ER with an asthma attack during this 7month period, 6 could not be approached for technical reasons and 250 were excluded for one of the following reasons: age $>55$ years (86 patients); $\mathrm{FEV}_{1}$ above $50 \%$ of predicted value (45 patients); history of chronic cough, emphysema or other chronic lung disease (43 patients); inability to perform the diagnostic tests proposed by the study (22 patients); pneumonia (21 patients); cardiac or other chronic diseases (12 patients); pregnancy (12 patients); absence of informed consent (5 patients), and consent withdrawn during the study (4 patients).

Of the 88 patients studied, $65(73.9 \%)$ were good responders and 23 (26.1\%) were poor responders. Of the 23 poor responders, 3 (3.4\% of all patients) required hospital admission and 20 (22.7\% of all patients) sought further emergency care for relapse of asthma within the next 10 days.

Table 1 lists the characteristics of the 88 patients studied and Table 2 lists the mean values for the variables analyzed (baseline and after $1 \mathrm{~h}$ of treatment).

Of the 30 variables studied, 6 were significantly different at the $\mathrm{P}<0.05$ level in the poor responder group compared to the good responder group (Table 2).

The multivariate analyses identified two independent variables that most contributed to the discrimination between groups: PEFR after $1 \mathrm{~h}(\mathrm{P}=0.01)$ and accessory muscle use after $1 \mathrm{~h}(\mathrm{P}=0.01)$.

We then selected these two predictive variables to develop a multifactorial index. The single cutoff point for each selected continuous variable that maximized predictive significance was obtained using a ROC curve.

Next, a predictive index using a combination of presenting factors was developed: PEFR after $1 \mathrm{~h} \leq 40 \%$ of the predicted value and 
accessory muscle use after $1 \mathrm{~h}$ of treatment. The index ranged from 0 to 2 , increasing with the severity of the measures. A comparison of index sensitivity, specificity, predictive values, and area under the ROC curve across different cutoff scores indicated that a cutoff score of 1 resulted in the least error of classification in differentiating poor responders from good responders (Figure 1). An index of 1 or higher presented 74.0 sensitivity, 69.0 specificity, 46.0 positive predictive value, and 88.0 negative predictive value.

Eighty-eight percent of the patients who had index scores of 0 were in the group of good responders and $12 \%$ of the patients with this score were in the group of poor responders. However, more of the patients who had an index score of 2 were in the poor responder group (75\%). When the index scores were 1 , there was a marked overlap between poor responders and good responders (Figure 2).

\section{Discussion}

The present results provide information on the current assessment of early outcome prediction of acute asthma in the ER setting. In agreement with previous studies $(3,12-$ $14)$, we found that no single clinical or pul-

Table 1. Demographic characteristics.

\begin{tabular}{|c|c|c|c|c|c|c|}
\hline Variables & Total & $\begin{array}{l}\text { Good } \\
\text { responders }\end{array}$ & $\begin{array}{c}\text { Poor } \\
\text { responders }\end{array}$ & $\mathrm{RR}$ & $95 \% \mathrm{Cl}$ & $\mathrm{P}$ \\
\hline \multicolumn{7}{|l|}{ Sex } \\
\hline Female & $71.6 \%$ & $43(66.2 \%)$ & $20(87.0 \%)$ & 2.64 & $(0.86-8.12)$ & 0.10 \\
\hline Male & $28.4 \%$ & $22(33.8 \%)$ & $3(13.0 \%)$ & & & \\
\hline Age (years) & $33 \pm 12$ & $33.24 \pm 12.07$ & $34.22 \pm 13.11$ & - & - & 0.75 \\
\hline Height (cm) & $163 \pm 10$ & $163.71 \pm 10.19$ & $162.17 \pm 10.7$ & - & - & 0.54 \\
\hline Weight (kg) & $68 \pm 15$ & $68.18 \pm 15.42$ & $69.32 \pm 15.59$ & - & - & 0.77 \\
\hline $\begin{array}{l}\text { Duration of the } \\
\text { attack prior to ER (h) }\end{array}$ & $34 \pm 46$ & $31.6 \pm 44.8$ & $40.78 \pm 50.8$ & - & - & 0.44 \\
\hline Smoker & $50.0 \%$ & $33(50.8 \%)$ & $11(47.8 \%)$ & 0.91 & $(0.45-1.85)$ & 0.99 \\
\hline Second-hand smoking & $45.3 \%$ & $28(43.8 \%)$ & $11(50 \%)$ & 1.20 & $(0.58-2.47)$ & 0.80 \\
\hline Regular medications & $44.3 \%$ & $28(43.1 \%)$ & $11(47.8 \%)$ & 1.15 & $(0.57-2.32)$ & 0.88 \\
\hline Short-acting $ß_{2}$-agonist & $23.0 \%$ & $15(23.4 \%)$ & $5(21.7 \%)$ & 0.93 & $(0.39-2.18)$ & 0.99 \\
\hline Long-acting ß2-agonist & $4.6 \%$ & $3(4.7 \%)$ & $1(4.3 \%)$ & 0.94 & $(0.16-5.34)$ & 0.99 \\
\hline Corticosteroids & $18.4 \%$ & $10(15.6 \%)$ & $6(26.1 \%)$ & 1.56 & (0.73-3.33) & 0.35 \\
\hline Inhaled & $11.5 \%$ & $8(12.5 \%)$ & $2(8.6 \%)$ & 0.73 & $(0.20-2.67)$ & 0.99 \\
\hline Oral & $6.9 \%$ & $2(3.1 \%)$ & $4(17.4 \%)$ & 2.84 & $(1.43-5.66)$ & 0.04 \\
\hline Methylxanthines & $13.8 \%$ & $7(10.9 \%)$ & $5(21.7 \%)$ & 1.73 & $(0.79-3.79)$ & 0.29 \\
\hline Ipratropium & $2.3 \%$ & $1(1.6 \%)$ & $1(4.3 \%)$ & 1.93 & $(0.46-8.08)$ & 0.46 \\
\hline Attack medications & $97.7 \%$ & $63(96.9 \%)$ & $23(100 \%)$ & - & - & 0.97 \\
\hline Short-acting $ß_{2}$-agonist & $93.1 \%$ & $58(90.6 \%)$ & $23(100 \%)$ & - & - & 0.33 \\
\hline Long-acting $ß_{2}$-agonist & $2.3 \%$ & $1(1.6 \%)$ & $1(4.3 \%)$ & 1.93 & $(0.46-8.08)$ & 0.46 \\
\hline Corticosteroids & $24.1 \%$ & $14(21.9 \%)$ & $7(30.4 \%)$ & 1.37 & $(0.65-2.88)$ & 0.59 \\
\hline Inhaled & $3.4 \%$ & $2(3.1 \%)$ & $1(4.3 \%)$ & 1.27 & $(0.25-6.56)$ & 0.99 \\
\hline Oral & $18.4 \%$ & $10(15.9 \%)$ & $6(26.1 \%)$ & 1.57 & $(0.74-3.34)$ & 0.35 \\
\hline Methylxanthines & $6.9 \%$ & $3(4.7 \%)$ & $3(13 \%)$ & 2.02 & $(0.83-4.91)$ & 0.19 \\
\hline Ipratropium & $21.8 \%$ & $12(18.8 \%)$ & $7(30.4 \%)$ & 1.56 & $(0.75-3.24)$ & 0.38 \\
\hline Length of ER stay (h) & $9 \pm 13$ & $6.74 \pm 5.37$ & $18.06 \pm 22.84$ & - & - & 0.06 \\
\hline
\end{tabular}

$\mathrm{RR}=$ relative risk for poor responders; $95 \% \mathrm{Cl}=95 \%$ confidence interval; $\mathrm{ER}=$ emergency room. 
Table 2. Clinical and pulmonary function measures obtained before and $1 \mathrm{~h}$ after treatment.

\begin{tabular}{|c|c|c|c|c|c|c|}
\hline Variables & Total & $\begin{array}{l}\text { Good } \\
\text { responders }\end{array}$ & $\begin{array}{c}\text { Poor } \\
\text { responders }\end{array}$ & $\mathrm{RR}$ & $95 \% \mathrm{Cl}$ & $P$ \\
\hline \multicolumn{7}{|l|}{ Dyspnea } \\
\hline Pretreatment & $94.3 \%$ & $62(95.4 \%)$ & $21(91.3 \%)$ & 0.63 & $(0.20-1.96)$ & 0.60 \\
\hline After $1 \mathrm{~h}$ & $44.3 \%$ & $24(36.9 \%)$ & $15(65.2 \%)$ & 2.35 & $(1.11-4.97)$ & 0.04 \\
\hline \multicolumn{7}{|l|}{ Cough } \\
\hline Pretreatment & $77.3 \%$ & $52(80 \%)$ & $16(69.6 \%)$ & 0.67 & $(0.32-1.40)$ & 0.46 \\
\hline After $1 \mathrm{~h}$ & $39.8 \%$ & $22(33.8 \%)$ & $13(56.5 \%)$ & 1.96 & $(0.97-3.98)$ & 0.10 \\
\hline \multicolumn{7}{|c|}{ Feeling of thoracic constriction } \\
\hline Pretreatment & $42.0 \%$ & 27 (41.5\%) & 10 (43.5\%) & 1.06 & $(0.52-2.15)$ & 0.99 \\
\hline After $1 \mathrm{~h}$ & $11.4 \%$ & $4(6.2 \%)$ & $6(26.1 \%)$ & 2.75 & $(1.42-5.31)$ & 0.02 \\
\hline \multicolumn{7}{|l|}{ Pulse rate (bpm) } \\
\hline Pretreatment & $102 \pm 16$ & $102.21 \pm 15.39$ & $101 \pm 19.14$ & - & - & 0.77 \\
\hline After $1 \mathrm{~h}$ & $107 \pm 19$ & $106.62 \pm 19.25$ & $106.23 \pm 19.27$ & - & - & 0.94 \\
\hline \multicolumn{7}{|c|}{ Respiratory rate (breath/min) } \\
\hline Pretreatment & $25 \pm 6$ & $24.35 \pm 5.38$ & $26.59 \pm 6.31$ & - & - & 0.12 \\
\hline After $1 \mathrm{~h}$ & $21 \pm 4$ & $20.38 \pm 4.14$ & $23.05 \pm 3.61$ & - & - & 0.009 \\
\hline \multicolumn{7}{|c|}{ Pulsus paradoxus } \\
\hline Pretreatment & $4.5 \%$ & $2(3.1 \%)$ & $2(8.7 \%)$ & 2.00 & $(0.70-5.71)$ & 0.28 \\
\hline After $1 \mathrm{~h}$ & $2.3 \%$ & $2(3.1 \%)$ & 0 & - & - & 0.99 \\
\hline \multicolumn{7}{|l|}{ Cyanosis } \\
\hline Pretreatment & $12.5 \%$ & $9(13.8 \%)$ & $2(8.7 \%)$ & 0.66 & $(0.18-2.45)$ & 0.72 \\
\hline After $1 \mathrm{~h}$ & $2.3 \%$ & $2(3.1 \%)$ & 0 & - & - & 0.99 \\
\hline \multicolumn{7}{|c|}{ Accessory muscle use } \\
\hline Pretreatment & $48.9 \%$ & $30(46.2 \%)$ & $13(56.5 \%)$ & 1.36 & $(0.66-2.76)$ & 0.54 \\
\hline After $1 \mathrm{~h}$ & $13.6 \%$ & $4(6.2 \%)$ & $8(34.8 \%)$ & 3.37 & (1.84-6.18) & 0.001 \\
\hline \multicolumn{7}{|c|}{ Supraclavicular or intercostal retractions } \\
\hline Pretreatment & $33.0 \%$ & $20(30.8 \%)$ & $9(39.1 \%)$ & 1.30 & $(0.64-2.65)$ & 0.63 \\
\hline After $1 \mathrm{~h}$ & $6.8 \%$ & $4(6.2 \%)$ & $2(8.7 \%)$ & 1.30 & $(0.39-4.27)$ & 0.65 \\
\hline \multicolumn{7}{|c|}{ Inability to speak } \\
\hline Pretreatment & $13.6 \%$ & $9(13.8 \%)$ & $3(13 \%)$ & 0.95 & $(0.33-2.71)$ & 0.99 \\
\hline After $1 \mathrm{~h}$ & $1.1 \%$ & $1(1.5 \%)$ & 0 & - & - & 0.99 \\
\hline \multicolumn{7}{|l|}{ Wheezing } \\
\hline Pretreatment & $95.5 \%$ & $63(96.9 \%)$ & $21(91.3 \%)$ & 0.50 & $(0.17-1.42)$ & 0.28 \\
\hline After $1 \mathrm{~h}$ & $69.3 \%$ & $43(66.2 \%)$ & $18(78.3 \%)$ & 1.59 & $(0.66-3.84)$ & 0.41 \\
\hline \multicolumn{7}{|c|}{ Oxygen saturation (\%) } \\
\hline Pretreatment & $94 \pm 3$ & $94.32 \pm 3.02$ & $94.87 \pm 1.87$ & - & - & 0.42 \\
\hline After $1 \mathrm{~h}$ & $95 \pm 2$ & $95.33 \pm 2.05$ & $94.37 \pm 2.34$ & - & - & 0.09 \\
\hline \multicolumn{7}{|l|}{$\mathrm{FEV}_{1}(\%)^{*}$} \\
\hline Pretreatment & $36 \pm 14$ & $37.23 \pm 14.87$ & $30.91 \pm 9.68$ & - & - & 0.02 \\
\hline After $1 \mathrm{~h}$ & $52 \pm 18$ & $55.82 \pm 18.10$ & $42.74 \pm 15.08$ & - & - & 0.003 \\
\hline \multicolumn{7}{|l|}{ PEFR $(\%)^{*}$} \\
\hline Pretreatment & $32 \pm 11$ & $33.43 \pm 10.40$ & $28.57 \pm 11.13$ & - & - & 0.06 \\
\hline After $1 \mathrm{~h}$ & $47 \pm 16$ & $50.43 \pm 15.23$ & $37.17 \pm 11.83$ & - & - & 0.000 \\
\hline \multicolumn{7}{|l|}{ FVC (\%)* } \\
\hline Pretreatment & $59 \pm 19$ & $60.57 \pm 20.16$ & $53.13 \pm 16.17$ & - & - & 0.11 \\
\hline After $1 \mathrm{~h}$ & $76 \pm 21$ & $78.06 \pm 19.18$ & $69.30 \pm 25.39$ & - & - & 0.09 \\
\hline
\end{tabular}

$\mathrm{RR}=$ relative risk for poor responders; $95 \% \mathrm{Cl}=95 \%$ confidence interval; $\mathrm{FEV}_{1}=$ forced expiratory volume in $1 \mathrm{~s}$; PEFR = peak expiratory flow rate; FVC = forced vital capacity.

*Predict for sex, race, age and height. 
monary functional factor assessed during the first hour of management in the ER could adequately predict the outcome of acute episodes. Previous studies have suggested that a multifactorial assessment of patients with acute asthma may provide the key to achieving this goal (8). So, we proposed an index score composed of two variables which are commonly and easily measured in the assessment of patients with acute asthma. Despite our efforts, wide overlap between good responders and poor responders was observed, with a predictor index score of 1 . On the other hand, a predictor index score of 0 suggested that the patient should be considered for discharge, while an index score of 2 suggested that the patient should be considered for prompt hospitalization. The fact that a substantial proportion of our patients had an index score of 1 emphasizes the need for additional evaluation of clinical data and lung function in predicting the post-discharge course and the need for admission to hospital.

Previous studies have demonstrated that the failure of initial therapy to improve expiratory flow predicts a more severe course and the need for hospitalization (15-17).

In 1976, Banner et al. (15) studied 67 episodes of acute asthma treated in an ER and suggested that severely obstructed patients (PEFR $<16 \%$ of predicted) whose PEFR remained less than $60 \mathrm{l} / \mathrm{min}$, or who exhibited a less than $16 \%$ improvement following 0.3 $\mathrm{ml}$ epinephrine be promptly admitted.

In 1979, Nowak et al. (18) studied 82 patients before and after emergency therapy for acute asthma. An $\mathrm{FEV}_{1} \leq 0.6$ liter before treatment or an $\mathrm{FEV}_{1} \leq 1.6$ liters after emergency treatment was associated with an unfavorable course. They concluded that spirometry could identify asthmatic patients who would require admission or who would have significant airway obstruction within $48 \mathrm{~h}$ after discharge from the ER.

In 1981, Fischl et al. (3) evaluated 205 patients who presented to the ER for treatment of asthma. A predictive index using a combination of presenting factors was developed: pulse rate $\geq 120 / \mathrm{min}$, respiratory rate $\geq 30 / \mathrm{min}$, pulsus paradoxus $\geq 18 \mathrm{mmHg}$, PEFR $\leq 120 \mathrm{l} / \mathrm{min}$, moderate to severe dyspnea, accessory muscle use, and wheezing. The index ranged from 0 to 7 , increasing with the severity of symptoms. An index of 4 or higher was $95 \%$ accurate in predicting the risk of relapse and $96 \%$ accurate in predicting the need for hospitalization.

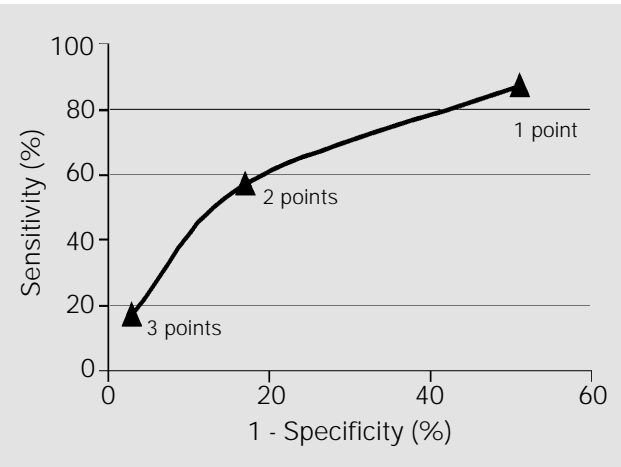

Figure 1. ROC curve obtained from the predictive index score.

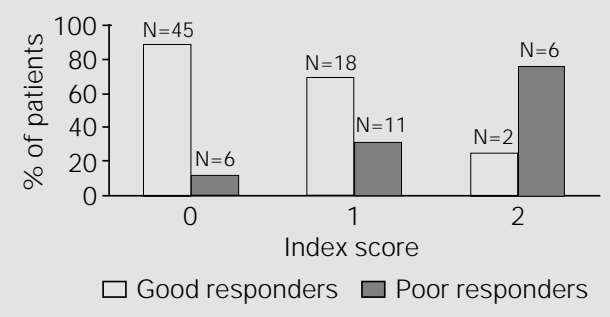

Figure 2. Index score for 88 patients with acute asthma.

Table 3. Comparison between the indices.

\begin{tabular}{lcccc}
\hline Index & Score 0 & Score 1 & Score 2 & $\begin{array}{c}\text { Total of } \\
\text { possible points }\end{array}$ \\
\hline $\begin{array}{l}\text { Present study } \\
\text { PEFR after } 1 \mathrm{~h} \leq 40 \% \text { predicted }\end{array}$ & $\begin{array}{c}\text { Absent } \\
\text { Absent }\end{array}$ & $\begin{array}{c}\text { Present } \\
\text { Present }\end{array}$ & - & 2 points \\
Accessory muscle use at $1 \mathrm{~h}$ & & & & \\
Rodrigo and Rodrigo (14) & & & & 4 points \\
PEFR at 30 min (\% predicted) & $\geq 50$ & $40-49$ & $<40$ & \\
Change in PEFR from baseline & $\geq 90$ & $60-89$ & $<60$ & \\
at 30 min (I/min) & & & & \\
Rodrigo and Rodrigo (13) & & & & 6 points \\
PEFR variation at 30 min (I/min) & $>50$ & $50-20$ & $<20$ & \\
PEFR at 30 min (\% predicted) & $>45$ & $45-35$ & $<35$ & \\
Accessory muscle use at 30 min & $0-1$ & 2 & 3 & \\
\hline
\end{tabular}

PEFR = peak expiratory flow rate. 
In 1992, Bolliger et al. (12) assessed 52 patients with acute asthma who presented to the ER. The patient's clinical data and lung function were recorded. In the cited study it was not possible to reliably predict on admission the long-term response of individual patients to treatment of an asthma attack with or without a predictive index. Applying the Fischl index (3) to the patients studied was even less useful than applying PEFR alone owing to a considerable overlap.

In 1997, Rodrigo and Rodrigo (13) examined 184 adults with acute asthma who presented to the ER. Discriminant analysis identified three independent variables that made the greatest contribution to the discrimination between discharged and hospitalized patients: PEFR variation over baseline, PEFR as percent of predicted value, and accessory muscle use, all measured $30 \mathrm{~min}$ after the beginning of treatment. The index presented 0.86 sensitivity, 0.96 specificity, 0.75 positive predictive value, and 0.98 negative predictive value.

In 1998, Rodrigo and Rodrigo (14) developed an index with only two variables: PEFR as percent of predicted value and PEFR variation over baseline, both measured over $30 \mathrm{~min}$. The index presented 0.79 sensitivity, 0.96 specificity, 0.94 positive predictive value, and 0.86 negative predictive value.

The main difference between our study and those of Rodrigo and Rodrigo $(13,14)$ is that we used a single cutoff point for each selected variable (PEFR after $1 \mathrm{~h} \leq 40 \%$ of predicted value and presence or absence of accessory muscle use after $1 \mathrm{~h}$ of treatment), in contrast with the use of two cutoff points for the selected variables in the cited studies (see Table 3). Also we used PEFR as percent of predicted value instead of PEFR variation over baseline. These modifications were intended to simplify the index score used in clinical practice.

Another difference was the treatment protocol: in our study albuterol was delivered with a continuous flow nebulizer at 20-min intervals for $60 \mathrm{~min}$ (three doses), as compared with albuterol delivered with a metered-dose inhaler into a spacer device activated at 10 -min intervals for $30 \mathrm{~min}$ (three doses) in the work of Rodrigo and Rodrigo $(13,14)$. As a result, in our study we developed a predictive index for evaluating patients after $60 \mathrm{~min}$ of treatment, as opposed to evaluation after $30 \mathrm{~min}$ of treatment in Rodrigo and Rodrigo's work $(13,14)$.

By contrast with these two studies $(13,14)$ we were unable to predict outcome reliably for our patients in the ER with or without a predictor index. These findings are in accordance with most earlier studies that failed to reliably identify poor outcome by measurements of single risk factors or by multifactorial predictor indices applied to acute asthmatic patients during their ER stay (12).

In our study, the treatment protocol and the clinical criteria used to discharge the asthmatic patients from the ER resulted in a $22.7 \%$ relapse rate within 10 days, similar to that reported by others, which ranged from 10 to $37 \%(3,13,18,19)$.

Measurement of airflow obstruction in acute asthma should be made using bedside determination of PEFR or FEV $\mathrm{F}_{1}$. In general, the results of these tests are expressed in relation to predicted values for sex, age and height. However, some asthmatic patients may have significant fixed airflow obstruction even when they are asymptomatic. Knowledge of the degree of fixed airflow obstruction during asymptomatic periods and the patient's personal best PEFR or $\mathrm{FEV}_{1}$ is useful in interpreting lung function measurements made during the acute exacerbation. Unfortunately, this information is usually not available in the acute setting $(4,7)$.

Current guidelines for the management of asthma recognize the importance of preventive anti-inflammatory therapy (6). Individuals who require emergency treatment for their asthma are generally considered to have extremely poorly controlled disease and to be at increased risk for death. It was 
found that patient education about the proper management of asthma can significantly reduce the number of asthma attacks and ER visits (20). In our study, only $11.5 \%$ of the asthmatic patients had been treated with inhaled corticosteroids in the ambulatory care setting. We believe that this finding reflects the low socioeconomic status of our asthmatic population and their inappropriate reliance on ER care. Patients with these characteristics would benefit from more intensive management of their asthma. Provision of self-management education in the ER and facilitating the referral and access to ambulatory care facilities from the ER might serve to reduce asthma morbidity (20).

In the present study it was possible to predict early outcome in the first hour of management of acute asthma in the ER setting when the index score was 0 or 2 . For the patients with an index score of 1 , significant overlap between good and poor outcome indicates the need for additional evaluation.

\section{Acknowledgments}

We are indebted to Prof. Mário Bernardes Wagner and to the statistician Vânia Naomi Hirakata for the statistical analyses, to the nurses Márcia Pires, Márcia Severo and Márcia Brambilla for technical assistance in the selection and treatment of the patients, and to all other members of the ER medical, administrative and nursing staff for their collaboration.

\section{References}

1. Smith DH, Weiss K \& Sullivan SD (2000). Epidemiology and costs of acute asthma. In: Hall J B, Corbridge TC, Rodrigo C \& Rodrigo GJ (Editors), Acute Asthma: Assessment and Management. McGrawHill, New York, NY, USA, 1-10.

2. Kelsen SG, Kelsen DP, Fleegler BF, J ones RC \& Rodman T (1982). Emergency room assessment and treatment of patients with acute asthma. American J ournal of Medicine, 72: 416-422.

3. Fischl MA, Pitchenik A\& Gardner LB (1981). An index predicting relapse and need for hospitalization in patients with acute bronchial asthma. New England J ournal of Medicine, 305: 783-789.

4. Corbridge TC \& Hall J B (1995). The assessment and management of adults with status asthmaticus. American J ournal of Respiratory and Critical Care Medicine, 151: 1296-1316.

5. Dalcin PTR, Medeiros $A C$, Siqueira $M K$, Mallmann F, Lacerda M, Gazzana MB \& Menna Barreto SS (2000). Acute asthma in adults in the emergency room: clinical management in the first hour. J ornal de Pneumologia, 26: 297-306.

6. National Heart, Lung, and Blood Institute (1997). Guidelines for the Diagnosis and Management of Asthma. NIH Publication No. 97-4051A, Bethesda, MD, USA.

7. Rodrigo GJ \& Rodrigo C (2000). Emergency department assessment: severity and outcome prediction. In: Hall J B, Corbridge TC, Rodrigo C \& Rodrigo GJ
(Editors), Acute Asthma: Assessment and Management. McGraw-Hill, New York, NY, USA, 125-138.

8. Rodrigo G \& Rodrigo C (1993). Assessment of the patient with acute asthma in the emergency room: a factor analytic study. Chest, 104: 1325-1328.

9. American Thoracic Society (1987). Standards for the diagnosis and care of patients with chronic obstructive pulmonary disease (COPD) and asthma. American Review of Respiratory Disease, 136: 225-244.

10. Knudsom RJ, Slatin RC, Lebowitz MD \& Burrow B (1976). The maximal expiratory flow-volume curve - normal standards, variability and effects of age. American Review of Respiratory Disease, 113: 587-600.

11. Dalcin PTR (1996). Estudo da depuração pulmonar do radioaerossol de dietilenotriaminopentacetato marcado com tecnécio$99 \mathrm{~m}$ no lúpus eritematoso sistêmico. Doctoral thesis, Curso de Pós-Graduação em Medicina-Pneumologia, Faculdade de Medicina, Universidade Federal do Rio Grande do Sul, Porto Alegre, RS, Brazil.

12. Bolliger $C T$, Fourie PR, Kotze $D$ \& J oubert J R (1992). Relation of measures of asthma severity and response to treatment to outcome in acute severe asthma. Thorax, 47: 943-947.

13. Rodrigo $G \&$ Rodrigo $C$ (1997). A new index for early prediction of hospitalization in patients with acute asthma. American J ournal of Emergency Medicine, 15: 8-13.
14. Rodrigo G \& Rodrigo C (1998). Early prediction of poor response in acute asthma patients in the emergency department. Chest, 114: 1016-1021.

15. Banner AS, Shah RS \& Addington WW (1976). Rapid prediction of need for hospitalization in acute asthma. J ournal of the American Medical Association, 235: 13371338.

16. Fanta $\mathrm{CH}$, Rossing $\mathrm{TH} \& \mathrm{M}$ cFadden J $r$ ED (1982). Emergency room treatment of asthma: relationships among therapeutics combinations, severity of obstruction and time course of response. American J ournal of Medicine, 72: 416-422.

17. Stein LM \& Cole RP (1990). Early administration of corticosteroids in emergency room treatment of acute asthma. Annals of Internal Medicine, 112: 822-827.

18. Nowak RM, Gordon RK, Wroblewski DA Tomlanovich MC \& Kvale PA (1979). Spirometric evaluation of acute bronchial asthma. J ournal of the American College of Emergency Physicians, 8: 9-12.

19. Fitzgerald J M \& Hargreave FE (1990). Acute asthma: emergency department management and prospective evaluation of outcome. Canadian Medical Association J ournal, 142: 591-595.

20. Hanania NA, David-Wang A, Kesten S \& Chapman KR (1997). Factors associated with emergency department dependence of patients with asthma. Chest, 111: 290295. 